\title{
Propriedades Psicométricas da Versão Reduzida da Escala de Percepção de Suporte Social
}

\author{
Verônica Morais Ximenes ${ }^{1}$ \\ Bárbara Barbosa Nepomuceno ${ }^{2}$ \\ James Ferreira Moura Jr. ${ }^{1}$ \\ Marcia Kelma de Alencar Abreu \\ Gabriela Oliveira Ribeiro ${ }^{7}$ \\ ${ }^{1}$ Universidade Federal do Ceará \\ ${ }^{2}$ Faculdade Ari de Sá
}

\section{Resumo}

O suporte social é um processo relacional no qual ajudas informacionais, instrumentais e afetivas são oferecidas por grupos e/ou pessoas, capaz de produzir efeitos positivos no bem-estar, na saúde e no estresse. O estudo avaliou as propriedades psicométricas da versão reduzida da Escala de Percepção de Suporte Social (EPSS) para pessoas que vivem em pobreza. Participaram da pesquisa 1.113 adultos (questionários válidos) com idade média de 42,25 anos ( $D P=17,57)$, homens (29,7\%) e mulheres (70,3\%), dos estados brasileiros Ceará, Amazonas e Paraná. Realizaram-se análises de confiabilidade, fatoriais exploratória e confirmatória. Verificou-se adequação de estrutura bifatorial do instrumento, além dos índices de confiabilidade e consistência interna. Os fatores apresentam satisfatórias cargas fatoriais e itens agrupados de maneira diferente da escala original. Originalmente compunha-se pelos fatores Suporte Prático e Suporte Emocional, propõe-se nova nomeação para Suporte Cognitivo-Instrumental e Suporte Emocional-Cooperativo. A versão reduzida da EPSS é adequada para populações que vivem em pobreza.

Palavras-chave: suporte social, psicometria, pobreza

\section{Psychometrics properties of the Reduced Version of the Social Support Perception Scale}

\begin{abstract}
Social support is a relational process in which informational, instrumental, and affective aids are offered by groups and / or persons, capable of producing positive effects on well-being, health, and stress. The study aimed to evaluate the psychometric properties of the reduced version of the Social Support Perception Scale (SSPS) for people living in poverty contexts. Participants comprised 1113 (valid questionnaires) adults with an average age of 42.25 years (SD = 17.57), including men (29.7\%) and women (70.3\%), from the Brazilian states of Ceará, Amazonas, and Paraná. Reliability, factorial exploratory and confirmatory analyzes were performed. The bifactorial structure of the instrument was verified, as well as excellent reliability and internal consistency indexes. Factors presented satisfactory factor loads and items grouped differently from the original scale. Originally composed by the factors Practical Support and Emotional Support, it is proposed a new nomenclature of factors such as Cognitive-Instrumental Support and Emotional-Cooperative Support. The reduced version of the SSPS is considered suitable for use in populations living in poverty contexts.
\end{abstract} Keywords: social support; psychometrics; poverty

\section{Propiedades psicométricas de la Versión Reducida de la Escala de Percepción de Soporte Social}

\section{Resumen}

El soporte social es un proceso relacional en el cual ayudas informativas, instrumentales y afectivas son ofrecidas por grupos y/o personas, con capacidad de producir efectos positivos en el bienestar, la salud y el estrés. La investigación tuvo como objetivo evaluar las propiedades psicométricas de la versión reducida de la Escala de Percepción de Soporte Social (EPSS) en personas que viven en situación de pobreza. Participaron de la investigación 1.113 (encuestas válidas) adultos con edad promedio 42,25 años ( $\mathrm{DP}=17.57)$, hombres $(29,7 \%)$ y mujeres $(70,3 \%)$, de los estados brasileños de Ceará, Amazonas y Paraná. Se realizaron análisis de confiabilidad, factoriales exploratoria y confirmatoria. Los resultados muestran adecuación de la estructura bifactorial del instrumento, además de los índices de confiabilidad y consistencia interna. Los factores presentan satisfactorias cargas factoriales e ítems agrupados de manera diferente de la escala original. Originalmente estaba compuesta por los factores Soporte Práctico y Soporte Emocional, y después se propuso el cambio de denominación para Soporte Cognitivo-Instrumental y Soporte Emocional-Cooperativo. Se considera que la versión reducida de la EPSS es adecuada para el uso en poblaciones que viven en la pobreza.

Palabras clave: soporte social; psicometría; pobreza 


\section{Introdução}

Suporte social e apoio social são termos utilizados em estudos para a análise das relações sociais, não existindo um consenso na literatura científica quanto à definição de tais conceitos, ora utilizados como sinônimos ora como termos que se aproximam (Gonçalves, Pawlowski, Bandeira, \& Piccinini, 2011). Neste estudo, opta-se por considerar os termos como sinônimos, uma vez que ambos são traduções possíveis do termo em inglês social support. $\mathrm{Na}$ análise do suporte social, é possível focar em diferentes perspectivas de análise, a saber: as fontes de suporte; os tipos de suporte; a intensidade e qualidade do suporte; a satisfação com suporte recebido; os efeitos do suporte tanto para quem recebe quanto para quem oferece apoio; e no mapeamento da rede de suporte disponível (Griep, Chor, Faerstein, Werneck, \& Lopes, 2005). Neste estudo, investigam-se os tipos de suporte social disponíveis, a partir da percepção do sujeito que recebe o suporte.

Para Rodrigues e Cohen (1998), o suporte social é um processo relacional no qual ajudas materiais e psicológicas são disponibilizadas aos sujeitos, capaz de promover efeitos positivos na saúde mental e física. Segundo Valla (1999), o suporte social gera efeitos emocionais e comportamentais positivos nos sujeitos envolvidos, ou seja, tanto em quem oferece apoio como em quem recebe. A partir de diferentes estudos, pode-se compreender que tal processo relacional é capaz de gerar efeitos positivos na saúde (Yunes \& Juliano, 2015), na diminuição do estresse (Gonçalves et al., 2011) e promover bem-estar (Andrade \& Vaitsman, 2002) aos sujeitos envolvidos.

No que se refere aos tipos de ajuda disponibilizadas, três tipos de suporte são os mais mencionados pelos estudos, a saber: o instrumental, o informacional e o emocional (Rodrigues \& Cohen, 1998; Chor, Griep, Lopes, \& Faerstein, 2001; Gonçalves et al., 2011) O suporte emocional envolve uma relação onde há confiança para a partilha e escuta de problemas vivenciados, assim como aconselhar, expressar gestos de carinho, preocupação e cuidado. O suporte instrumental envolve as ajudas práticas importantes na solução de problemas, tais como o cuidado com crianças e familiares, empréstimo de materiais diversos, possibilitar o transporte ou deslocamento, provir alimentação ou remédios. E o terceiro, o suporte informacional, envolve a obtenção de informações/orientações úteis para a solução de situações ou problemáticas vividas. Ademais desses tipos, suporte afetivo (demonstrações físicas de afeto), suporte cognitivo (postura ativa de incentivo, escuta, importante para a autoafirmação), interação social positiva (ter companhia para realizar atividades prazerosas) e participação também são tipos de suporte social mencionados em diferentes estudos (Griep et al., 2005).

No Brasil, podem-se encontrar diferentes instrumentos que se propõe a avaliar o suporte social. Entre eles destacam-se: Escala de Apoio Social do Estudo Pró-Saúde, uma validação e adaptação da escala norte-americana Social Support Survey of the Medical Outcomes Study (Griep et al., 2005); a Social Support Inventory for People who are Positive or Have AIDS, adaptada e validada por Seidl e Tróccoli (2006); o Social Support Questionnaire validado por Matsukura, Marturano e Oishi (2002); a Escala de Percepção de Suporte Social, validada por Baptista, Baptista e Torres (2006); a Escala de Percepção de Suporte Social construída por Cardoso e Baptista (2014); o Questionário de Suporte Social de Norbeck, traduzido por Andriola, Tróccoli e Dias (1990); a Escala de Percepção de Suporte Social criada no Brasil por Siqueira (2008); a Escala Multidimensional da Suporte Social Percebido, traduzida e validada por Gabardo-Martins, Ferreira e Valentini (2017); Escala de Satisfação com o Suporte Social que passou por validação transcultural Brasil-Portugal por Marôco, Campos, Vinagre e Pais-Ribeiro (2014), dentre outros. Tais instrumentos possibilitam uma avaliação do suporte social em função dos tipos de apoio recebidos, da identificação da origem e da intensidade e da satisfação com o apoio recebido.

A Escala de Percepção de Suporte Social (EPSS), instrumento avaliado neste artigo, foi elaborada por Siqueira (2008) e tem o objetivo de avaliar a rede de suporte social percebida pelos sujeitos, é voltada para o estudo das relações sociais de forma ampla, sem limitação a um contexto específico, a partir de uma avaliação psicossocial do suporte social. A autora se sustenta na conceituação de suporte social de Bowling (1997) como um processo interativo promotor de ajudas instrumentais e emocionais, e se centra nos principais tipos de suporte definidos por Rodriguez e Cohen (1998): instrumental, informacional e emocional. $\mathrm{O}$ instrumento não limita sua aplicação a um contexto específico e/ou perfil específico de sujeitos, mas foi validado para amostra formada prioritariamente por mulheres universitárias. Além de apresentar adequados indicadores psicométricos (Siqueira, 2008), os autores compreendem que a escala apresenta linguagem de simples compreensão para sujeitos de diferentes níveis de escolaridade, o que 
se enquadra de forma satisfatória em estudos desenvolvidos com pessoas em situação de pobreza.

Pesquisas brasileiras utilizaram a EPSS em diferentes contextos e sujeitos, a saber: Ribeiro, Siqueira e Claro (2012) avaliam o suporte social como um indicador de saúde positiva em expatriados; Gomes e Oliveira (2013) analisaram a relação entre depressão e ansiedade à percepção de suporte social em enfermeiros; Pereira e Resende (2014) investigam o suporte social como protetivo à saúde e redutor do estresse em pacientes idosos portadores de doença arterial coronariana; Villela avalia a correlação entre uso de álcool e aspectos sociocognitivos em diferentes tratamentos para doença renal crônica; e Bezerra-Ribeiro (2008) investiga a percepção de suporte social e o consumo de álcool em desempregados.

De acordo com Gonçalves, Pawlowski, Bandeira e Piccinini (2011), o suporte social tem sido central nos estudos que focam o enfrentamento às situações estressoras e desafiadoras vividas pelos sujeitos, configurando-se como um importante recurso individual e coletivo. Pesquisas evidenciam o suporte social como uma importante estratégia coletiva de sobrevivência empregada por pessoas que vivem em situação de pobreza e vulnerabilidade social (Nepomuceno, Silva, \& Ximenes, 2016; Dutra, Prates, Nakamura, \& Villela, 2013), já que possibilita aos sujeitos dispor de recursos materiais, instrumentais e afetivos para lidar com as dificuldades diárias (Lever \& Martínez, 2007). Ademais, ao considerar os atuais processos sociais de atomização dos indivíduos e desintegração social, vigentes nas sociedades contemporâneas, o estudo das relações de suporte é de fundamental importância, pois podem ser indicadores de fatores de risco (situações de baixo apoio) ou fatores de proteção (situações de alto índice de apoio) (Andrade \& Vaitsman, 2002).

As pessoas em situação de pobreza têm uma trajetória de vida marcada por questões sociais e simbólicas adversas e específicas, originadas pela desigualdade social, que repercutem em uma constituição psíquica singular (Samman, 2009). Atesta-se que o suporte social é central para indivíduos inseridos nesse contexto, pois funciona como uma estratégia de sobrevivência quando há a prevalência da situação de desemprego (Teschl \& Comim, 2005); as políticas governamentais são insuficientes para a garantia dos direitos sociais básicos (Rego \& Pinzani, 2013); e as organizações não governamentais não são capazes de fornecer os diferentes tipos de apoios (informacional, instrumental e afetivo) necessários a uma vida digna (Sen, 2013).
O desenvolvimento de instrumentais capazes de contribuir com a avaliação e caracterização dos contextos de pobreza se faz relevante, uma vez que a problemática da pobreza é presente na vida de milhões brasileiros. Segundo o Instituto Brasileiro de Geografia e Estatística, em 2016, aproximadamente 50 milhões de brasileiros, $25,4 \%$ da população, viviam na linha de pobreza, com renda familiar equivalente a US $\$ 5,5$ por dia (definição de pobreza do Banco Mundial) ou R \$387,07 (IBGE, 2017). É importante salientar que, em contextos de crise política e econômica, há uma tendência de aumento dos índices de pobreza no Brasil (Banco Mundial, 2017).

Estudos nacionais e internacionais revelam que tal problemática deve ser considerada a partir de uma perspectiva multidimensional, superando análises e medições reducionistas e unidimensionais restritas à esfera das privações materiais. De acordo com Sen (2010), pobreza envolve a privação de capacidades básicas dos sujeitos, o acesso a bens e serviços, participação política, questões de gênero, dentre outras. A análise e enfretamento da problemática deve considerar os aspectos culturais e relacionais envolvidos, captando o lugar social ocupado por sujeitos pobres e os recursos coletivos e individuais de enfrentamento estabelecidos. Nesse processo, há formas de sociabilidade basais para a dinâmica de vida das pessoas em situação de pobreza, sendo as relações de suporte sociais centrais nas estratégias de sobrevivência (Rego \& Pinzani, 2013).

Analisando os estudos desenvolvidos com a escala, observa-se a necessidade do desenvolvimento de uma versão reduzida adaptada para pessoas em situação de pobreza. Portanto, é importante elaborar instrumentos voltados para contextos locais em situação de pobreza, com atenção especial às formas de estruturação dos instrumentos, pois escalas com uma quantidade extensa de itens não são atrativas para os respondentes, devendo ser priorizadas as versões reduzidas (Esteban-Guitart \& Sánchez-Vidal, 2012). Assim, este estudo objetiva avaliar as propriedades psicométricas da versão reduzida da Escala de Percepção de Suporte Social (EPSS) (Siqueira, 2008) para pessoas que vivem em contextos de pobreza. Para tanto, são examinadas as evidências da adequação psicométrica da versão reduzida da escala.

\section{Método}

\section{Participantes}

Participou da pesquisa um total de 1.156 pessoas de ambos os sexos. Desse total, 1.113 questionários 
foram válidos, o que configurou a exclusão de 43 participantes, a partir do critério não ter respondido de maneira integral a Escala de Percepção de Suporte Social. Considerando os questionários validados, a amostra foi comporta por sujeitos moradores de comunidades rurais dos Estados do Amazonas $(\mathrm{N}=$ 376), Paraná $(\mathrm{N}=361)$ e Ceará $(\mathrm{N}=376)$, dos quais 70,3 \% são mulheres, possuem idade média de 42,25 anos (DP $=17,57) ; 53,1 \%$ estão desempregados; $36,9 \%$ estão no mercado informal e 10\% têm vínculo empregatício; 39,4\% nunca estudaram; 34,4\% têm até cinco anos de estudo; $16,9 \%$, de seis a nove anos de estudo e 9,3\%, mais de 10 anos; 29,8\% relatam já ter deixado de utilizar algum serviço de saúde por não ter dinheiro para pagar o transporte." Em relação à moradia, $57,4 \%$ moravam em casa de tijolo, os demais em casas feitas com outros materiais; apenas $73,7 \%$ possuem banheiro com vaso sanitário e descarga; $17,7 \%$, com vaso sanitário sem descarga; 5,5\% banheiro com buraco no chão e 3,1\% não possuem banheiro. Em relação à renda per capita familiar, 33,3\% possuem renda de até $1 / 4$ do salário mínimo ${ }^{1} ; 31,8 \%$ até $1 / 2$ salário mínimo; $23,2 \%$ até um(1) salário mínimo e $11,7 \%$ maior que um(1) salário mínimo.

\section{Instrumento}

Foram utilizadas variáveis referentes à caracterização da pobreza, como escolaridade, empregabilidade, acesso à saúde, condições de moradia e renda. Essas variáveis serviram para caraterização da amostra. Também, foi utilizada a Escala de Percepção de Suporte Social (EPSS) que é composta por 29 itens, distribuídos em dois fatores, método de extração dos eixos principais com rotação oblimin. O primeiro fator é Suporte prático, com 19 itens, relativos ao apoio instrumental e informacional (alfa de Cronbach $=0,91,39,66 \%$ de variância comum, autovalor de 11,50 com cargas fatoriais variando de 0,38 a 0,78$)$. O segundo fator é Suporte emocional, com 10 itens (alfa de Cronbach $=$ 0,92, 4,02\% da variância comum, autovalor de 1,17 com cargas fatoriais entre $-0,55$ a $-0,83$ ) (Siqueira, 2008). Neste estudo de validação, a amostra contava com 437 participantes, sendo $84,2 \%$ estudantes universitários. O KMO foi identificado com 0,953, e o Teste de Esferacidade de Bartlett's teve um índice significativo $\left(\chi^{2}=\right.$ 6467.225; $g l=406 ; p<0,01)$.

\footnotetext{
Considerando o salário mínimo vigente no período da coleta de dados no valor de R \$880,00, no ano de 2016.
}

Para o presente estudo, foi realizada uma redução da escala. Tal adaptação consistiu em uma redução do instrumento para 12 itens, dos quais oito são relativos ao suporte prático (quatro informacional e quatro instrumental) e quatro ao suporte emocional (ver Tabela 01). Tais itens da escala pontuam de 0 a $3(0=$ Nunca; 1 = Poucas vezes; 2 = Muitas vezes; 3 = Sempre) como possibilidades de resposta.

\section{Procedimentos de Coleta}

Em respeito aos parâmetros e itens que regem a Resolução 466, de 2012, do Conselho Nacional de Saúde do Brasil, a pesquisa foi submetida ao Comitê de Ética em Pesquisa, sendo aprovada com CAAE: 46474715.5.1001.5054 e Parecer $\mathrm{N}^{\circ}$ 1.233.648. A pesquisa foi realizada em contextos com altos índices de vulnerabilidade social de acordo com as instituições parceiras que atuaram no desenvolvimento da investigação. Essas parcerias ocorreram com universidades, Centros de Referência de Assistência Social e Unidades Básicas de Saúde nas cidades. Foram aplicados os questionários nos estados do Paraná, Amazonas e Ceará. Os domicílios foram indicados segundo as avalições de profissionais dessas instituições que atuavam nas regiões pesquisadas. $\mathrm{O}$ instrumento foi aplicado de forma individual com a leitura do questionário por um pesquisador, no domicílio dos sujeitos ou nas próprias instituições parceiras.

\section{Procedimentos de Análise dos Dados}

Sobre as análises realizadas, informa-se que são assumidos índices adequados de normalidade, linearidade e homocedasticidade da amostra. Foram realizadas análises de correlação entre os itens da escala. Foi executada análise fatorial exploratória (AFE) com utilização do Método de Componentes Principais, sendo adotado o ponto de corte de 0,30 de carga fatorial para inclusão na estrutura dos fatores. E, igualmente, foram efetuadas análises fatoriais confirmatórias (AFC) com estrutura fatorial da escala original e o modelo advindo da AFE. Para realização dessas análises, como os casos perdidos representavam menos $5 \%$ da amostra, foi realizada a imputação de valores para proceder com a realização da AFC. Foi utilizado como método da AFC a estimação da máxima verossimilhança (Díaz et al., 2006). Os índices de ajustes aceitáveis foram: o Índice Comparativo de Ajuste (CFI) e o Índice de Tucker e Lewis (TLI) superiores a 0,95 ; a raiz quadrada média do erro de aproximação (RMSEA) (McDonald \& Ho, 2002) inferior a 0,05 ; e a raiz quadrada média residual 
padronizada (SRMR) inferior a 0,05 (Hair, Black, Babin, \& Tatham 2009).

\section{Resultados}

Os itens da escala foram escolhidos considerando a maior adequação ao contexto social, econômico e cultural de pessoas em situações de pobreza e a carga fatorial de cada item. Esse procedimento baseia-se nas considerações de Hair, Black, Babin e Tatham (2009) que informam que as maiores cargas fatoriais indicam os itens mais representativos do fator. A Tabela 1 apresenta os itens selecionados e suas cargas fatoriais na escala original.

Ao realizar análises de correlação entre os itens das variáveis da Escala Reduzida de Percepção de Suporte Social (Tabela 2), foram identificadas correlações positivas e elevadas em todos os itens da versão reduzida. Identifica-se, assim, uma excelente relação entre os itens constituintes da escala.

Após as correlações, foi realizado Método de Componentes Principais da análise fatorial exploratória (AFE) com rotação oblimin direta. Foram assumidas pressuposições de esfericidade e homocedasticidade $\left[\mathrm{KMO}=0,924\right.$, Teste de Esfericidade de Barlett $\chi^{2}(66)$ $=5817,986, p<0,001]$. AFE indicou que a escala foi estruturada em dois fatores com eigenvalues acima de
1,00 , sendo o fator $1 \mathrm{com}$ autovalor 5,93 e o fator 2 com autovalor 1,09 (Figura 1).

A variância explicada total da escala reduzida é $58,61 \%$. É importante apontar que os itens não seguiram a organização dos fatores que estavam estabelecidos na proposta original da escala. No entanto, identifica-se que os itens se agruparam de uma maneira que baseou a proposição de novos fatores explicativos da Escala Reduzida de Percepção de Suporte Social (Tabela 3).

Aponta-se que os itens foram agrupados com elevadas cargas fatoriais entre $-0,860$ e 0,579 , indicando uma boa composição de fatores. Indica-se que o único item que obteve uma baixa carga fatorial foi "Substitua você em tarefas que não pode realizar no momento". Além disso, esse item tem carga fatorial compartilhada com os dois fatores, mas, por conta da fundamentação teórica, percebe-se como sendo esse item constituinte do fator 2. Seguindo essa explicação teórica, observa-se que o fator 1 pode ser intitulado como Suporte Cognitivo-Instrumental. Já o fator 2 pode ser chamado de Suporte Emocional-Cooperativo.

Igualmente, foi realizada análise de confiabilidade da escala. Identificou-se que o instrumento possui um excelente índice de confiabilidade com alfa de Cronbach $=0,905$. Também, foram realizadas análises de confiabilidade dos fatores identificados na redução da escala, sendo verificados igualmente bons índices. $O$ fator 1 tem 0,869 de alfa, já o fator 2 possui 0,825 . Como

Tabela 1.

Cargas Fatoriais e Comunalidades $\left(H^{2}\right)$ dos Itens Selecionados da EPSS Original

\begin{tabular}{|c|c|c|c|}
\hline Item & $\begin{array}{l}\text { Suporte } \\
\text { Prático }\end{array}$ & $\begin{array}{c}\text { Suporte } \\
\text { Emocional }\end{array}$ & $\mathrm{h}^{2}$ \\
\hline Empreste algo que você precisa. & 0,68 & & 0,466 \\
\hline Dê sugestões sobre seu futuro. & 0,58 & & 0,468 \\
\hline Empreste dinheiro a você. & 0,58 & & 0,401 \\
\hline Oriente suas decisões. & 0,53 & & 0,510 \\
\hline Substitua você em tarefas que não pode realizar no momento. & 0,53 & & 0,330 \\
\hline Forneça alimentação quando você precisa. & 0,50 & & 0,285 \\
\hline Esclareça as suas dúvidas. & 0,44 & & 0,420 \\
\hline Ajude você a resolver um problema prático. & 0,44 & & 0,388 \\
\hline Ouve com atenção seus problemas pessoais. & & $-0,80$ & 0,675 \\
\hline Compreenda suas dificuldades. & & $-0,76$ & 0,535 \\
\hline Esteja ao seu lado em qualquer situação. & & $-0,70$ & 0,563 \\
\hline Preocupa-se com você. & & $-0,62$ & 0,545 \\
\hline
\end{tabular}

Fonte: adaptado de Siqueira (2008). 
Tabela 2.

Correlações entre os Itens da Versão Reduzida da EPSS

\begin{tabular}{|c|c|c|c|c|c|c|c|c|c|c|c|c|}
\hline & 1 & 2 & 3 & 4 & 5 & 6 & 7 & 8 & 9 & 10 & 11 & 12 \\
\hline $\begin{array}{l}\text { 1. Empreste algo } \\
\text { que você precisa. }\end{array}$ & 1 & & & & & & & & & & & \\
\hline $\begin{array}{l}\text { 2. Dê sugestões } \\
\text { sobre seu futuro. }\end{array}$ & $.407^{* *}$ & 1 & & & & & & & & & & \\
\hline $\begin{array}{l}\text { 3. Ouve com } \\
\text { atenção seus } \\
\text { problemas } \\
\text { pessoais. }\end{array}$ & $.375^{* *}$ & $.578^{* *}$ & 1 & & & & & & & & & \\
\hline $\begin{array}{l}\text { 4. Empreste } \\
\text { dinheiro a você. }\end{array}$ & $.578^{* *}$ & $.414 * *$ & $.403^{* *}$ & 1 & & & & & & & & \\
\hline $\begin{array}{l}\text { 5. Oriente suas } \\
\text { decisões. }\end{array}$ & $.397 * *$ & $.575^{* *}$ & $.543^{* *}$ & $.451^{* *}$ & 1 & & & & & & & \\
\hline $\begin{array}{l}\text { 6. Compreenda } \\
\text { suas dificuldades. }\end{array}$ & $.389 * *$ & $.454 * *$ & $.542^{* *}$ & $.473^{* *}$ & $.651^{* *}$ & 1 & & & & & & \\
\hline $\begin{array}{l}\text { 7. Substitua você } \\
\text { em tarefas que } \\
\text { não pode realizar } \\
\text { no momento. }\end{array}$ & $.300 * *$ & $.382 * *$ & $.351 * *$ & $.367 * *$ & $.422 * *$ & $.482^{* *}$ & 1 & & & & & \\
\hline $\begin{array}{l}\text { 8. Esclareça as } \\
\text { suas dúvidas. }\end{array}$ & $.370^{* *}$ & $.494 * *$ & $.516^{* *}$ & $.410^{* *}$ & $.602^{* *}$ & $.590 * *$ & $.540^{* *}$ & 1 & & & & \\
\hline $\begin{array}{l}\text { 9. Esteja ao seu } \\
\text { lado em qualquer } \\
\text { situação. }\end{array}$ & $.357 * *$ & $.341 * *$ & $.422 * *$ & $.349 * *$ & $.432 * *$ & $.498^{* *}$ & $.398^{* *}$ & $.461 * *$ & 1 & & & \\
\hline $\begin{array}{l}\text { 10. Forneça } \\
\text { alimentação } \\
\text { quando você } \\
\text { precisa. }\end{array}$ & $.336^{* *}$ & $.355^{* *}$ & $.323^{* *}$ & $.423^{* *}$ & $.423^{* *}$ & $.422^{* *}$ & $.378^{* *}$ & $.425^{* *}$ & $.528^{* *}$ & 1 & & \\
\hline $\begin{array}{l}\text { 11. Ajude você } \\
\text { a resolver um } \\
\text { problema prático. }\end{array}$ & $.363^{* *}$ & $.395^{* *}$ & $.404 * *$ & $.403^{* *}$ & $.467 * *$ & $.525^{* *}$ & $.470 * *$ & $.556^{* *}$ & $.535^{* *}$ & $.598 * *$ & 1 & \\
\hline $\begin{array}{l}\text { 12. Preocupa-se } \\
\text { com você. }\end{array}$ & $.311^{* *}$ & $.355^{* *}$ & $.410^{* *}$ & $.318^{* *}$ & $.406^{* *}$ & $.460 * *$ & $.383^{* *}$ & $.430^{* *}$ & $.611^{* *}$ & $.514^{* *}$ & $.568^{* *}$ & 1 \\
\hline
\end{tabular}

Fonte: Elaborada pelos autores.

$* * p<0,001$

forma de testar o modelo original reduzido e a nova proposta de divisão de fatores identificada na pesquisa, foi efetuada também uma análise fatorial confirmatória (AFC) com o modelo original e com a estrutura fatorial advinda da AFE da Escala Reduzida de Percepção de Suporte Social.

Analisando os índices da AFC (Tabela 4), identifica-se que o modelo mesmo com inclusão de duas covariâncias não obteve índices satisfatórios do CFI
$(0,897)$ e do TLI $(0,897)$, ficando abaixo de 0,90 . O RMSEA está distante do valor indicado como adequado com 0,093(IC90\% = 0,086-0,101). O único índice que seria considerado bom é o SRMR $(0,0487)$, pois está abaixo de 0,05 . Apesar disso, essa disposição de índices não contribui para uma validação do ajuste do modelo original.

Assim, procedeu-se com a realização de uma AFC com o modelo identificado na análise fatorial 


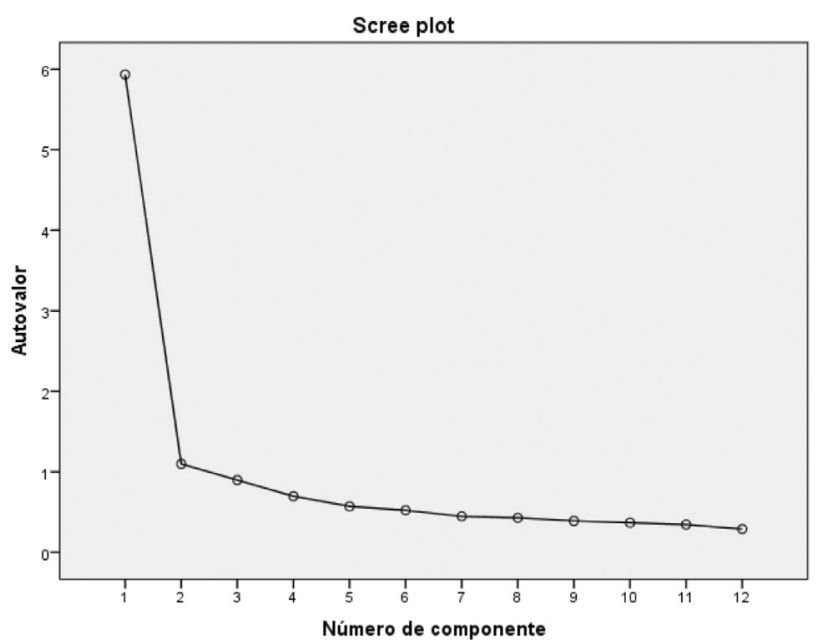

Fonte: Elaborada pelos autores.

Figura 1. Scree Plot dos autovalores da análise fatorial exploratória da versão reduzida da EPSS. exploratória da escala reduzida de Percepção de Suporte Social. Dessa maneira, os resultados da AFC para a Escala Reduzida de Percepção de Suporte Social indicam que o modelo final obteve índices de ajustes adequados com a inclusão de duas covariâncias (Tabela 5). Assim, identificou-se que o modelo melhorou seus índices com a inclusão da covariância entre os erros dos itens "Esclareça as suas dúvidas" e "Substitua você em tarefas que não pode realizar no momento" e dos itens "Empreste dinheiro a você" e "Empreste algo que você precisa". O CFI porta um índice excelente com 0,989; o RMSEA $(0,067 / \mathrm{IC} 90 \%=0,059-0,075)$ e SRMR $(0,0378)$ estão adequados aos índices mais robustos propostos por Hair et al., (2009). O TLI $(0,947)$ também possui um bom índice próximo ao apontado como adequado. É importante salientar que os índices de ajustes verificados são adequados, apresentando valores acima daqueles considerados robustos, para confirmação dos construtos mensurados (Byrne, 2010).

Tabela 3.

Análise de Componentes Principais da Escala Redu乏ida de Percepção de Suporte Social

\begin{tabular}{lcc}
\hline Itens & Fator & Fator \\
& 1 & 2 \\
\hline
\end{tabular}

Suporte Cognitivo - instrumental

$\begin{array}{ll}\text { Empreste algo que você precisa. } & .718\end{array}$

$\begin{array}{ll}\text { Dê sugestões sobre seu futuro. } & .817\end{array}$

$\begin{array}{ll}\text { Ouve com atenção seus problemas pessoais. } & \text {.702 }\end{array}$

$\begin{array}{ll}\text { Empreste dinheiro a você. } & .743\end{array}$

$\begin{array}{ll}\text { Oriente suas decisões. } & .760\end{array}$

Compreenda suas dificuldades. $\quad .621$

$\begin{array}{ll}\text { Esclareça as suas dúvidas. } & .579\end{array}$

Suporte Emocional - Cooperativo

Substitua você em tarefas que não pode realizar no momento.

Esteja ao seu lado em qualquer situação.

Forneça alimentação quando você precisa.

Ajude você a resolver um problema prático.

Preocupa-se com você.

Confiabilidade (alfa de Cronbach) 
Tabela 4.

Índices de Ajuste da AFC da Versão Reduzida da EPSS como Estrutura Original de Fatores

\begin{tabular}{lccccccc}
\hline & $\chi^{2}$ & $\boldsymbol{D f}$ & $\boldsymbol{P}$ & TLI & CFI & RMSEA (C.I.) & SRMR \\
\hline Modelo Inicial & 783.229 & 53 & $<.001$ & .843 & .874 & $.116(.109-.123)$ & .0595 \\
Modelo Modificado com 2 covariâncias & 508.810 & 51 & $<.001$ & .897 & .897 & $.093(.086-.101)$ & .0487 \\
\hline
\end{tabular}

Fonte: Elaborada pelos autores.

Tabela 5.

Indices de Ajuste da AFC da Versão Reduzida da EPSS como Nova Estrutura de Fatores

\begin{tabular}{llllllll}
\hline & $\chi^{2}$ & Df & $\boldsymbol{P}$ & TLI & CFI & RMSEA (C.I.) & SRMR \\
\hline Modelo Inicial & 489.583 & 53 & $<.001$ & .906 & .943 & $.089(.082-.097)$ & .0490 \\
Modelo Modificado com 2 covariâncias & 285.742 & 51 & $<.001$ & .947 & .989 & $.067(.059-.075)$ & .0378 \\
\hline
\end{tabular}

Fonte: Elaborada pelos autores

\section{Discussão}

Considerando o objetivo do estudo de avaliar as propriedades psicométricas da versão reduzida da Escala de Percepção de Suporte Social (EPSS) para pessoas que vivem em contextos de pobreza, observa-se que a versão reduzida alcançou bons índices psicométricos de validação de sua adaptação para populações em situação de pobreza. De acordo com Pilatti, Pedroso e Gutierrez (2010), para alcançar uma boa consistência interna, o instrumento deve ter uma correlação moderada entre os itens. Observa-se que a versão reduzida da EPSS obteve essas correlações moderadas indicadas.

Identifica-se que a análise fatorial exploratória apresentou a Escala Reduzida de Suporte Social com uma estrutura bifatorial, diferente da estrutura dos itens da escala original. Apesar disso, a variância total explicada e as cargas fatoriais dos itens apresentam bons índices de validade, comparando com a versão original de Siqueira (2008). De acordo com Field (2009), os itens devem possuir cargas fatoriais maiores que 0,35 nos fatores que são correspondentes, representando assim uma medida válida e precisa. É importante evidenciar que as cargas fatoriais da maioria dos itens da versão reduzida da escala de EPSS estão entre 0,57 e 0,82 , tendo somente um item com carga abaixo desses valores.

A nova estrutura fatorial, com cargas fatoriais estatisticamente consistentes, reafirma e reitera a importância de se trabalhar a partir de uma escala adaptada para esse novo público, cujas características requerem a utilização de um instrumento que considere as implicações psicossociais da pobreza (Moura Jr., Cidade, Ximenes, \& Sarriera, 2014). Para tanto, necessita-se reinterpretar teoricamente os novos fatores revelados à luz das características da amostra trabalhada, o que incita a necessidade de articulação das especificidades da população em situação de pobreza.

Destarte, traz-se a proposição de dois fatores: Suporte Cognitivo-Instrumental e Suporte Emocional-Cooperativo, constituindo duas dimensões relevantes da percepção de suporte social para o público trabalhado nessa validação, já que representam perspectivas do apoio que podem estar relacionadas ao enfrentamento das implicações psicossociais da pobreza em uma perspectiva multidimensional. Griep, Chor, Faerstein, Werneck e Lopes (2005) também apresentam o apoio material em uma dimensão separada do apoio emocional, por considerar a diferença entre esses dois aspectos.

Dessa maneira, compreende-se que o fator Suporte Cognitivo-Instrumental refere-se a auxílios que envolvem a autoafirmação, uma postura ativa de incentivo, escuta e reforço positivo, assim como o fornecimento de instrumentais importantes à resolução de problemas ou realização de uma atividade (Gonçalves et al., 2011). Os itens relacionados a esse fator estariam vinculados a um esforço cognitivo das pessoas que dariam suporte ao indivíduo em situação de pobreza. Além disso, esses itens podem ser 
reconhecidos como atividades práticas que também estão presentes nos atos de emprestar dinheiro e algo que se precisa. As pessoas em situação de pobreza têm uma demanda por atividades práticas que estão vinculadas às suas problemáticas cotidianas.

Considerar a realidade de privação multidimensionalidade da pobreza (Sen, 2010), superando os vieses de leitura limitados às restrições financeiras e materiais, põe em evidência a necessidade de observar o fenômeno em suas expressões socioeconômicas, políticas, culturais, simbólicas e relacionais. Considerando tal dimensão relacional, o instrumento de percepção de suporte social possibilita a identificação dos tipos de ajudas disponíveis percebidas pelos sujeitos em condição de pobreza. Dessa maneira, uma das dimensões centrais do construto suporte social são as características referentes a uma atuação ativa de apoio na resolução de problemáticas concretas, de ordem instrumental/material, informacional e cognitiva, como aquelas envolvendo o fator Suporte Cognitivo-Instrumental constituinte da versão reduzida.

Igualmente, entre as privações relacionadas à realidade de vulnerabilidade social também está presente o processo de estigmatização da pobreza, que causa sentimentos de vergonha e atitudes de isolamento social em pessoas inseridas nessa situação (Zavaleta, 2007). Dessa maneira, é importante, além de questões práticas relacionadas ao suporte instrumental e cognitivo, a existência de relações de apoio baseadas na afetividade.

Assim, o fator Suporte Emocional-Cooperativo abrangeria esse âmbito das trocas afetivas constituintes das relações de suporte, porque envolve a percepção de ser cuidado, apoiado e valorizado positivamente por alguém emocionalmente (Gonçalves et al., 2011), assim como o envolvimento ativo de outra pessoa na realização conjunta de atividades ou resolução de problemáticas. Segundo Cruz (2016), o afeto, constituinte das relações sociais, é importante elemento para a superação do sofrimento causado pelas dificuldades próprias ao contexto de pobreza e capazes de fortalecer as redes de solidariedade em uma postura ativa frente à realidade social. Assim, os itens vinculados a esse fator são reconhecidos para além de um suporte cognitivo-instrumental, são ações voltadas para uma disposição por inteiro a ajudar com ações que correspondem uma vinculação emocional. Essas ações estão presentes de forma evidente nas comunidades em situação de pobreza, com o fortalecimento das redes de apoio e de afeto para lidar com as adversidades do contexto
(Guardiola, Gonzalez-Gomez, Garcia-Rubio, \& Lendechy-Grajales, 2012).

A dimensão cooperativa do fator anuncia um tipo de ajuda que implica uma ação coletiva, na qual ambas as partes (quem oferece e quem recebe o apoio) estão ativamente envolvidas na resolução da problemática ou superação da dificuldade. Segundo Ávila-Toscano (2009), é a partir das interações recíprocas estabelecidas entre as pessoas que se instaura uma rede de entregas sociais representadas através dos favores oferecidos como expressão do suporte social. Diz ainda o autor, que tal interação é produtora de efeitos nos planos afetivos e materiais.

Fornecer alimento, por exemplo, tipo de ajuda presente em um dos itens do fator, caracteriza um suporte que envolve uma questão concreta de nutrição do corpo e simultaneamente é permeada por significações simbólicas das práticas sociais com implicações no plano emocional. Não é uma relação de troca instrumental como emprestar dinheiro em que se baseia no retorno financeiro do valor provido. Fornecer alimento é uma doação de tempo e de cuidado para o indivíduo que necessita de alimentação por conta da situação de pobreza e, consequentemente, de fome (Rego, \& Pinzani, 2013). Isso se deve ao fato de os alimentos, culturalmente, serem considerados não só a partir do seu valor nutricional, mas também de sua importância simbólica e emocional. Para Romanelli (2006), a alimentação possui uma dimensão afetiva, que envolve a relação com o outro, momentos de trocas interpessoais, conversas, de estabelecimento de relações sociais. De forma similar, os estudos realizados por Griep et al., (2005) também consideram as dimensões afetiva e interação social positiva como complementares, já que o fato de construir coletivamente pode implicar em uma relação mútua de afetos, constituindo-se como aspectos integrantes de uma mesma perspectiva de suporte social.

De acordo com Hair et al., (2009), na análise de confiabilidade, o valor do alfa de Cronbach deve ser mínimo 0,70 , sendo baseada na consistência interna a partir da variância dos componentes individuais e da variância da soma dos componentes. É importante salientar que o índice da escala e dos fatores está entre 0,75 e 0,90 , indicando uma consistência alta de acordo com Cronbach e Meehl (1955). Os valores são semelhantes aos estudos de Pereira e Resende (2014) e Siqueira (2008).

Verifica-se que a análise fatorial confirmatória corroborou com a estrutura da Escala Reduzida 
de Percepção de Suporte Social advinda da AFE, obtendo índices muito superiores a estrutura original de fatores da escala, o que põe em evidência a boa adequação da versão proposta da escala para sua utilização com pessoas em situação de pobreza. Considerando que o público para o qual a redução da escala se destina apresenta características específicas, no que se refere à sociabilidade, afetividade, comunicação, portanto à percepção de suporte social, os dados trouxeram novos elementos e novas compreensões para interpretar a estrutura fatorial da Escala Reduzida de Suporte Social.

Dessa maneira, compreende-se que a versão reduzida da escala voltada para pessoas em situação de pobreza estrutura-se de uma maneira que contempla as especificidades dos tipos de suporte social mais recorridos nesse contexto. A mesma avalia o suporte em função dos tipos de apoio cognitivo-instrumental e emocional-cooperativo, contemplando diferentes perspectivas de percepção de suporte social necessárias ao enfrentamento das adversidades no âmbito das diversas necessidades concretas de suporte social, assim como das implicações psicossociais e afetivas forjadas na construção coletiva de fortalecimento das redes de solidariedade.

\section{Considerações Finais}

Destaca-se a relevância metodológica com o compromisso ético-político da Psicologia ao colaborar na adaptação e aperfeiçoamentos de instrumentos psicométricos para populações em contexto de pobreza. Salienta-se também a especificidade da dinâmica psicossocial desse contexto e seu impacto na constituição do suporte social, configurando-se como mecanismo de enfrentamento das limitações advindas da vulnerabilidade social.

A Escala de Percepção de Suporte Social (EPSS) na versão reduzia alcançou bons índices psicométricos de validação de sua adaptação, sendo adequada para o uso em contextos de pobreza e vulnerabilidade social. Ela estruturou-se de maneira específica para essa população, diferenciando-se da versão original, a partir de novos fatores voltados para aspectos cognitivo-instrumentais e emocional-cooperativos. Este último se refere às redes de apoio integral e afetivo em que se baseiam as trocas sociais estabelecidas em contextos de pobreza. O fator Cognitivo-Instrumental refere-se às ajudas de ordem instrumental/material, informacional e cognitivo.
A EPSS se limita à avaliação dos tipos de suporte percebidos pelos sujeitos, sendo necessária a utilização de outros instrumentos para uma exploração mais ampla e abrangente do suporte social, como: mapeamento da rede de suporte social disponível, as fontes de suporte, sua qualidade, sua intensidade e efeitos. Sugere-se a realização de futuras pesquisas que realizem a avaliação do instrumento na sua versão reduzida em outros Estados e Regiões brasileiras não incluídas neste estudo, assim como em comunidades urbanas. Destaca-se, também, a necessidade da validação de outras escalas voltadas para populações em situação de pobreza, devido à escassez de escalas próprias para esse coletivo.

\section{Referências}

Andrade, G. R. B., \& Vaitsman, J. (2002). Apoio social e redes: Conectando solidariedade e saúde. Ciência \& Saúde Coletiva, 7(4), 925-934. doi: 10.1590/ S1413-81232002000400023

Andriola W. B., Troccoli B. T., \& Dias, M. R. (1990) Caracterização do apoio social em estudantes universitários brasileiros. Rev Psicol; 7(8), 61-78. doi: 10.1590/S1413-81232011000300012

Ávila-Toscano, J. H. (2009). Redes sociales, generación de apoyo sociais ante la pobreza y calidad de vida. Revista Iberoamericana de Psicología: ciencia y tecnologia, 2 (2), 65-73. Recuperado de https://dialnet.unirioja. es/descarga/articulo/4905171.pdf

Banco Mundial. (2017). Salvaguardas contra a reversão dos ganhos sociais durante a crise econômica no Brasil. Poverty and Inequality Monitoring: Latin America and the Caribbean. Recuperado de https:// nacoesunidas.org/wp-content/uploads/2017/02/ NovosPobresBrasil_Portuguese.pdf

Baptista, M. N., Baptista, A. S. D., \& Torres, E. C. R. (2006). Associação entre suporte social, depressão e ansiedade em gestantes. Revista de Psicologia da Vetor Editora, 7(1), 39-48. Recuperado de http:// pepsic.bvsalud.org/pdf/psic/v7n1/v7n1a06.pdf

Bezerra-Ribeiro, M. (2008). Percepção de suporte social e consumo de álcool em desempregados (Dissertação de mestrado não publicada). Mestrado em Psicologia da Saúde. Universidade Metodista de São Paulo, São Bernardo do Campo, Brasil.

Bowling, A. (1997). Measuring health: A review of quality of life measurement scales. Philadelphia: Open University.

Psico-USF, Bragança Paulista, v. 25, n. 2, p. 371-383, abr./jun. 2020 
Byrne, B. M. (2010). Structural equation modeling with AMOS: Basic concepts, applications, and programming (2nd ed.). New York, NY: Routledge.

Cardoso, H. F., \& Baptista, M. N. (2014). Escala de Percepção do Suporte Social (versão adulta) - EPSUS-A: Estudo das qualidades psicométricas. Psico-USF [online], 19(3), 499-510. doi: 10.1590/1413-82712014019003012

Chor, D., Griep, R. H., Lopes, C. S., \& Faerstein, E. (2001). Medidas de rede e apoio social no estudo pró-saúde: Pré-testes e estudo piloto. Cadernos de Saúde Pública, 17(4), 887-896. doi: 10.1590/ S0102-311X2001000400022

Cronbach, L. J., \& Meehl, P. E. (1955). Construct validity in psychological tests. Psychological Bulletin, 52(4), 281-302.

Cruz, J. M. (2016). Sentidos dos afetos das famílias em situação de pobreza acompanbadas pelo CRAS (Dissertação de mestrado não publicada). Universidade Federal do Ceará, Fortaleza, Brasil.

Díaz, D., Rodríguez-Carvajal, R., Blanco, A., Moreno-Jiménez, B., Gallardo, I., Valle, C., \& Van Dierendonck, D. (2006). Adaptación española de las escalas de bienestar psicológico. Psicothema, 18, 572-577. Recuperado de http://www.crecimientopositivo.es/Materiales/well-being-psycothema.pdf

Dutra, M. D. L., Prates, P. L., Nakamura, E., \& Villela, W. V. (2013). A configuração da rede social de mulheres em situação de violência doméstica. Ciência \& Saúde Coletiva, 18(5), 1293-1304. doi: 10.1590/ S1413-81232013000500014

Esteban-Guitart, M., \& Sánchez-Vidal, A. (2012). Sentido de comunidad en jóvenes indígenas y mestizos de San Cristóbal de las Casas (Chiapas, México). Un estudio empírico. Anales de Psicología, 28(2), 532-540. Recuperado de http://www.redalyc.org/ pdf/167/16723135024.pdf

Gabardo-Martins, L. M. D., Ferreira, M. C., \& Valentini, F. (2017). Propriedades psicométricas da escala multidimensional de suporte social percebido. Temas em Psicologia, 25(4), 1873-1883. doi: 10.9788/ TP2017.4-18Pt

Gomes, R. K., \& Oliveira, V. B. (2013). Depressão, ansiedade e suporte social em profissionais de enfermagem. Boletim de Psicologia, 63(138), 23-33. Recuperado de http://pepsic. bvsalud.org/scielo.php?script=sci_arttext\&pid =S0006-59432013000100004

Gonçalves, T. R., Pawlowski, J., Bandeira, D. R., \& Piccinini, C. A. (2011). Avaliação de apoio social em estudos brasileiros: Aspectos conceituais e instrumentos. Ciência \& Saúde Coletiva, 16(3), 1755-1769. doi: 10.1590/S1413-81232011000300012

Griep, R. H., Chor, D., Faerstein, E., Werneck, G. L., \& Lopes, C. S. (2005). Validade de constructo de escala de apoio social do Medical Outcomes Study adaptada para o português no Estudo Pró-Saúde. Saúde. Cad Saúde Pública, 21(3), 703-14. doi: 10.1590/S0102-311X2005000300004

Guardiola, J., Gonzalez-Gomez, F., Garcia-Rubio, M. A., \& Lendechy-Grajales, A. (2012). Does higher income equal higher levels of happiness in every society? The case of the Mayan people. International Journal of Social Welfare, 22(1), 35-44. doi: 10.1111/j.1468-2397.2011.00857.x

Field, A. (2009). Descobrindo a estatística usando o SPSS. Porto Alegre: Artmed.

Hair, J. F., Black, W. C., Babin, B. J., Anderson, R. E., \& Tatham, R. L. (2009). Análise multivariada de dados. Porto Alegre: Bookman.

Instituto Brasileiro de Geografia e Estatística (IBGE) (2017). Pesquisa Nacional por Amostra de Domicílios Contínua 2016. Recuperado de https://biblioteca. ibge.gov.br/visualizacao/livros/liv101459.pdf

Lever, J. P., \& Martínez, Y. I. C. (2007). Pobreza y apoyo social: Un estudio comparativo en tres niveles socioeconómicos. Interamerican Journal of Psychology, 41(2), 177-188. Recuperado de http://www.redalyc.org/articulo.oa?id $=28441208$

Matsukura T. S., Marturano E. M., \& Oishi J. (2002). O Questionário de Suporte Social (SSQ): Estudos da adaptação para o português. Rev Latinoam Enferm, 10(5), 675-681. doi: 10.1590/ S0104-11692002000500008

Marôco, J. P., Campos, J. A. D. B., Vinagre, M. da G., \& Pais-Ribeiro, J. L. (2014). Adaptação Transcultural Brasil-Portugal da Escala de Satisfação com o Suporte Social para Estudantes do Ensino Superior. Psicologia: Reflexão e Crítica, 27(2), 247-256. doi: 10.1590/1678-7153.201427205

McDonald, R. P., \& Ho, M. R. (2002). Principles and practice in reporting structural equation 
analyses. Psychological Methods, 7(1), 64-82. doi: 10.1037//1082-989X.7.1.64

Moura Jr., J. F., Cidade, E. C., Ximenes, V. M., \& Sarriera, J. C. (2014). Concepções de pobreza: Um convite à discussão psicossocial. Temas em Psicologia, 22(2), 341-352. doi: 10.9788/TP2014.2-06

Nepomuceno, B. B., Silva, L. B., \& Ximenes, V. M. (2016). Estratégias de enfrentamento à pobreza: Uma análise a partir de estudos realizados com pessoas em sofrimento psíquico e prostitutas. Em V. M. Ximenes, B. B. Nepomuceno, E. C. Cidade \& J. F. Moura Jr. Implicações Psicossociais da Pobreza: Diversidades e Resistências (pp. 337-368). Fortaleza: Expressão Gráfica e Editora.

Pereira, I. A., \& Resende, M. C. (2014). Suporte social e depressão em idosos com doença arterial coronariana. Perspectivas em Psicologia, 18 (1), 97111. Recuperado de http://www.seer.ufu.br/ index.php/perspectivasempsicologia/article/ viewFile/28844/16026

Pilatti, L. A, Pedroso, B., \& Gutierrez, G. L. (2010). Propriedades Psicométricas de Instrumentos de Avaliação: Um debate necessário. Revista Brasileira de Ensino, Ciência e Tecnologia, 3(1), 81-91. doi: 10.3895/S1982-873X2010000100005

Rego, W., \& Pinzani, A. (2013). Vozes do Bolsa Família: Autonomia, dinheiro e cidadania. São Paulo: UNESP.

Ribeiro, P. E., Siqueira, M. M., \& Claro, J. A. C. D. S. (2012). Indicadores de saúde positiva: Um estudo com empregados expatriados. RAD - Revista Administração Em Diálogo, 14(1), 32-55. doi: 10.20946/ rad.v14i1.10180

Rodriguez, M. S., \& Cohen, S. (1998). Social support. Encyclopedia of Mental Health, 3, 535-544.

Romanelli, G. (2006). O significado da alimentação na família: Uma visão antropológica. Revista Faculdade de Medicina (Ribeirão Preto), 39(3), 333-339. Recuperado de http://www.revistas.usp.br/rmrp/article/ view/388/389

Samman, E. (2009). Una propuesta de indicadores internacionalmente comparables sobre bienestar psicológico y bienestar subjetivo. Las dimensiones faltantes de la pobreza. Oxford Poverty \& Human Development Initiative,
OPHI. Recuperado de http://www.ophi.org.uk/ wp-content/uploads/Missing_Dimensions_Shortlist_Psych_Subjective_Wellbeing_July09_sp.pdf

Seidl, E. M. F., \& Tróccoli, B. T. (2006). Desenvolvimento de escala para avaliação do suporte social em HIV/aids. Psicologia: Teoria E Pesquisa, 22(3), 317326. doi: 10.1590/S0102-37722006000300008

Sen, A. (2013). What's happening in Bangladesh? The Lancet, 382, 1966-1968. doi: 10.1016/

Sen, A. (2010). Desenvolvimento como liberdade. São Paulo: Companhia das Letras.

Siqueira, M. M. M. (2008). Construção e validação da Escala de Percepção de Suporte Social. Psicologia em Estudo, 13(2), 381-388. doi: 10.1590/ S1413-73722008000200021

Teschl, M., \& Comim, F. (2005). Adaptive preferences and capabilities: Some preliminary conceptual explorations. Review of Social Economy, 63(2), 229-247. doi: 10.1080/00346760500130374

Valla, V. (1999). Educação popular, saúde comunitária e apoio social numa conjuntura de globalização. Cadernos de Saúde Pública, v.15, S7-S14. doi:10.1590/ S0102-311X1999000600002

Villela, R. A. N. D. (2014). Relação entre o padrão de uso de álcool e saúde mental em pacientes com doença renal crônica. (Dissertação de Mestrado, Instituto de Ciências Humanas, Universidade Federal de Juiz de Fora, Minas Gerais). Recuperado de https:// repositorio.ufjf.br/jspui/handle/ufjf/769

Yunes, M. A. M., \& Juliano, M. C. C. (2015). A importância de redes de apoio na promoção da saúde e resiliência comunitária. Em J. C. Sarriera, E. T. Saforcada, \& J. L. Alfaro. (Orgs) Perspectiva psicossocial na saúde comunitária. (pp. 217-238). Porto Alegre: Sulina.

Zavaleta, D. R. (2007). The Ability to go about without shame: A proposal for internationally comparable indicators. OPHI Working Paper 03. Recuperado de http:/ /www.ophi.org.uk/wp-content/uploads/ OPHI-wp03.pdf

Recebido em: 07/08/2017 Reformulado em: 14/06/2018 
Sobre os autores:

Verônica Morais Ximenes é doutora em Psicologia pela Universidade de Barcelona (UB), pós-doutora em Psicologia pela Universidade Federal do Rio Grande do Sul (UFRGS), professora titular da Graduação e Pós-Graduação em Psicologia do Departamento de Psicologia da Universidade Federal do Ceará (UFC), coordenadora do Núcleo de Psicologia Comunitária (NUCOM) e bolsista de Produtividade em Pesquisa do CNPq PQ-2.

ORCID: https://orcid.org/0000-0003-3564-8555

E-mail:vemorais@yahoo.com.br

Bárbara Barbosa Nepomuceno é Doutora em Psicologia, mestre em Psicologia pela Universidade Federal do Ceará, master oficial em Intevención Psicossocial pela Universidad de Barcelona e graduada em Psicologia pela Universidade Federal do Ceará, docente do curso de Psicologia da Faculdade Ari de Sá (Fortaleza/CE) e integrante do Núcleo de Psicologia Comunitária da Universidade Federal do Ceará.

ORCID: https://orcid.org/0000-0002-1369-3040

E-mail: bbnepomuceno@yahoo.com.br

James Ferreira Moura Jr. é professor da Universidade da Integração Internacional da Lusofonia Afro-brasileira (UNILAB) e do Programa de Pós-graduação em Psicologia da Universidade Federal do Ceará (UFC), doutor em Psicologia pela UFRGS e coordenador da Rede de Estudos e Afrontamentos das Pobrezas, Discriminações e Resistências (REAPODERE).

ORCID: https://orcid.org/0000-0003-0595-5861

E-mail: james.mourajr@unilab.edu.br

Marcia Kelma de Alencar Abreu é Doutora em Psicologia pela Universidade Federal do Ceará (UFC) e no Programa Especial de Formação Pedagógica pela Universidade Estadual do Ceará, mestre em Psicologia pela UFC, doutoranda em Psicologia pela UFC, professora assistente da Universidade Regional do Cariri e integrante Núcleo de Psicologia Comunitária da UFC.

ORCID: https://orcid.org/0000-0002-2262-7918

E-mail:kelmabreu@yahoo.com.br

Gabriela Oliveira Ribeiro é psicóloga graduada pela Universidade Federal do Ceará.

ORCID: https://orcid.org/0000-0001-8161-0211

E-mail: gabiihribeiro22@gmail.com

Contato com os autores:

Profa. Verônica Morais Ximenes

Universidade Federal do Ceará - Departamento de Psicologia - Núcleo de Psicologia Comunitária - NUCOM

Av. da Universidade, 2762 - Benfica

Fortaleza-CE, Brasil

CEP: 60020-181

Psico-USF, Bragança Paulista, v. 25, n. 2, p. 371-383, abr./jun. 2020 
